\title{
An integrated framework for linking climate change impacts to emergency adaptation strategies for transport networks
}

\author{
Evangelos Mitsakis - Anestis Papanikolaou - Georgia Ayfadopoulou • \\ Josep Salanova • Claus Doll • George Giannopoulos • Christos Zerefos
}

Received: 16 July 2012 / Accepted: 23 July 2013 /Published online: 25 August 2013

(C) The Author(s) 2013. This article is published with open access at SpringerLink.com

\begin{abstract}
Introduction The assessment of the impacts of climate change and extreme weather events on transport networks comprises an emerging field, which attracts the interest of several researchers. Most research efforts have concentrated on the identification of adaptation measures and best practices for specific, local events and their impacts. Therefore, the present paper aims to fill in the gap of the current research and methodological activities in linking the impact assessment of climate change to emergency adaptation strategies for transport networks within an aggregated framework and thus linking the problem with its potential solutions.

Methods Following a targeted review of the state-of-practice and the relevant literature, a sequential concept for determining the optimal strategies for the relevant authorities is presented, comprised of five distinct dimensions: the extreme events, the area extent where extreme events have an impact on, the transport networks as separate modes, the transport networks as mode-internal components and finally the emergencyadaptation plans.

Results Each problem dimension is discussed both autonomously and as part of the sequential concept. A mathematical formulation for the integrated framework is presented, which
\end{abstract}

E. Mitsakis $(\bowtie) \cdot$ A. Papanikolaou $\cdot$ G. Ayfadopoulou $\cdot$ J. Salanova Centre for Research and Technology Hellas, Hellenic Institute of Transport, 6th km Charilaou-Thermi Rd.,

Thermi-Thessaloniki 57001, Greece

e-mail: emit@certh.gr

C. Doll

Institute for Systems and Innovation Research ISI, Breslauer Strasse 48, 76139, Karlsruhe, Germany

G. Giannopoulos

Hellenic Institute of Transport,

6th km Charilaou-Thermi Rd., Thermi-Thessaloniki 57001, Greece

C. Zerefos

Academy of Athens, Omirou 24, Athens 10564, Greece can be used by any interested entity (e.g. related authorities or organizations). The main theoretical aspects of the framework are also discussed in detail.

Conclusions The integrated top-down approach proposed can effectively address all the major dimensions for determining optimal adaptation strategies in a sequential way. The framework can address real world questions and issues about determining the appropriate combination of measures, in order to achieve the minimization of the economic losses due to potential extreme weather events with limited available resources.

Keywords Integrated framework - Emergency adaptation strategies $\cdot$ Climate change

\section{Introduction}

Over the last years great attention has been paid to the assessment of climate change and extreme weather events' impacts on the society, on several economic sectors and on transportation networks in particular. It is broadly acknowledged that transport constitutes a special field of the overall economic activity. Its role in the economy differs from other fields, due to the fact that transport is not an end in itself, but usually constitutes the means for satisfying other needs. The demand for transport is therefore considered as derived demand, occurring due to activities in other economic sectors or societal needs. For this reason - and since transport holds a critical part of the economic activities in any society - the impacts of climate change on transport networks entail serious losses and damages in all sectors, impairing eventually social stability. This is the main reason behind the realization of numerous studies, aiming to assess the impacts of extreme weather events on transport networks. Nevertheless, no unified approach has been considered so far, capable of defining accurately all the different parameters within one aggregated method and thus linking the problem with its potential solutions. 
The general objective of the majority of existing studies has been to identify the best practices or the appropriate adaptation measures for dealing with specific, local events. The current paper aims to provide a theoretical description and to contribute with a detailed identification of the entire series of questions that emerge, in order to accurately determine the optimal adaptation measures for addressing extreme weather events on transport networks.

\section{State-of-practice and literature review}

\subsection{Climate change policies at international level}

At a European Union level, in the recently published White Paper "Adapting to climate change: Towards a European framework for action" [1], the consequences of climate changes are identified as difficult for food supply, health, industry, ecosystems' integrity and the transport sector. In order to avoid climate change and the resulting consequences, two different directions are proposed: reducing greenhouse gas emissions (mitigation actions) and preparing for the unavoidable impacts (adaptation actions). Mitigation actions are taken in the form of various environmental policies, but it is recognized that more than 50 years will be necessary in order to see the policies' results. The White Paper recognizes that today's approach is disaggregated and highlights the need for a more aggregated approach. In the field of transport, the rise of sea level is defined as the worst impact of climate change for the infrastructures, especially at coastal areas. Although three scale levels are identified (national, regional and local), the EU level is proposed in the coordination procedures due to its strengthened role and characteristic of transcending boundaries of individual countries. The EU is working with other partner countries in the United Nations Framework Convention on Climate Change towards a post-2012 climate agreement, which will address both mitigation and adaptation. Different steps are programmed, including the creation of adaptation tools and a cost-benefit analysis for the year 2011. With the EU paper "Adapting Climate Change and Water, Coasts and Marine Issues" [2], accompanying the mentioned White Paper, special emphasis is placed by the EU on the impacts and measures for adaptation to climate change. Relevant information is presented in the "Impact assessment" paper [3], also accompanying the White Paper. This paper recognizes the potential impacts of climate change in Europe, presents a review of national adaptation strategies, including their costs and the benefits, together with a discussion on the adaptive capacity for infrastructures.

The economic impacts of climate change in Europe have been recently reviewed also by the EEA and the OECD [4]. These two reports have indicated that little quantified information exists on the costs and benefits of adaptation and that most studies focus on a few sectors, only by taking into account a limited sub-set of possible climate change effects.

The potential synergies or conflicts of mitigation and adaptation policies have been reviewed by Hamin and Gurran [5], focusing on US-based and Australian case studies. The authors identified that only half of the actions designed to address climate change are complementary to each other. Hunt's and Watkiss' [6] review on climate change impacts and the adaptation of cities, examined the progress of various cities concerning the quantification of climate change impacts, with the focus placed at one European (London) and one US megacity (New York). Their main findings deal with the limited up-to-date studies of quantified climate change risks and response to these risks at a city level, and the rather rarely addressed transport sector in these studies. Lovea's et al. dedicated research [7] on the linkage of climate change and variability with transportation, is also mainly oriented towards the European and US contexts. It culminated in the need to shift from "global impact assessment to finer regional and local-scale studies". They also purport that given adequate local scale datasets on extreme weather events, derived from long term monitoring and detection mechanisms, the uncertainties of events' probability occurrence can diminish.

\subsection{Performance of transport networks and criticality of network components}

The performance of transport networks and the criticality of network components have been studied by several researchers. Knoop et al. [8], suggest that vulnerability describes the weakness of a network, while criticality stands for the importance of its components. Taylor and D'Este [9] have proposed a methodology for obtaining the vulnerability of each component of the network, applicable on the national network level. The authors emphasize the differences between reliability and vulnerability: reliability is related to the connectivity of the network, while vulnerability is related to the consequences of failure. In their model, nodes are vulnerable and links have criticality values. An application of the proposed method on the Australian road network is also presented. Jenelius [10] and Knoop et al. [11] approaches deal with blocking every component of a network for determining their vulnerability. Single link failures and their impacts on networks have also been evaluated through various models in the studies of Taylor et al. [12], Jenelius et al. [13], Sohn [14] and Erath et al. [15]. Kim and Lee [16] identify the critical infrastructures from an economic point of view, reflecting the spatio-temporal characteristics of the economy. Nagurney and Qiang [17] propose a methodology for calculating criticality of network links, using the total demand of the network and 
the difference in the travel time as consequence of the closure of a link. The application of their proposed methodology is presented on the Sioux Falls network. The notion of network vulnerability in relation to extreme events is presented in a relevant research study of Schulz [18]. The author proposes not to act in the structural vulnerability, as "there is no way to reduce the hazard or it is unknown (e.g. for terrorist attacks) and constructions are already built and maintained in an optimal way" but to act in the functional vulnerability, defined as the consequential degree and duration of capacity reduction. The author applies the proposed method, in order to identify vulnerable locations of the road infrastructure in a German state, taking the passengers on each link as the potential damage and using the methodology proposed by Nagurney and Qiang [17] for calculating the criticality of each link. Other methods and metrics for quantifying network components' criticality are presented by MurrayTuite and Mahmassani [19], Chen et al. [20] and Sullivan et al. [21]

\subsection{Impact assessment of climate change and extreme events} on transport networks

In the study of Eichhorst [22] a list of events and related impacts on transport infrastructures is presented, together with adaptation measures proposed for several transport modes (road, rail, waterways). The author proposes also a sevenstep framework for developing an adaptation strategy for urban transport development, defining tools and guidelines for decision-making, which encompass step-by-step guides, wiki-based information portals, checklists and several other methods. Additionally, several studies are concerned with the quantification of the impacts of extreme events on transport network infrastructures. Relevant indices coupled with case study results from an urban agglomeration are presented in Suarez et al. [23], while empirical findings on the impacts of climate change and weather events on transport are presented in Koetse and Rietveld [24].

\subsection{Emergency management}

A number of definitions of 'emergency' and 'disaster' have been proposed over time, many of them focusing on some measure of the cost of the event in terms of loss of life or damage. However, the Emergency Management Australia Report [25] has underlined that the focus of concern with emergencies and disasters has moved towards consideration of the situation created by such phenomena rather than simply of the origin, nature, size, speed of onset and other physical attributes of the hazard, which result in the event itself. In 2007, the US Federal Emergency Management Agency [26] began an initiative which led to a wide international mobilization of emergency management experts, practitioners and academics, in order to consider principles of emergency management. This initiative was prompted by the realization that although numerous books, articles and papers referred to "principles of emergency management", the vast array of literature on the subject did not agree upon a common definition of these principles. The group agreed on eight principles to be used for guiding the development of a doctrine of emergency management, namely comprehensive, progressive, risk driven, integrated, collaborative, coordinated, flexible and professional.

\subsection{Research gaps and contributions of the paper}

A major finding, which highlights the need for the methodology proposed in the present paper, is the lack of the consideration of the geographic area extent. No relevant scientific literature or other studies exist, at least to the authors' knowledge, which include the geographic area extent within the existing assessment frameworks proposed by various researchers until today. This is one of the methodological and research gaps that the current paper aims to provide contribution to and incentives for further research activities. Furthermore, the literature review presented in this paper has indicated a gap of integrated approaches and methodologies for assessing the impacts of climate change related to emergency management adaptation strategies in a holistic way. A recent method, which attempts to correlate extreme weather events with socio-economic scenarios in a holistic approach, has been presented by Jaroszweski et al.[27].

\section{Foreword of the sequential concept for determining the optimal strategies}

\subsection{The multidimensional character of the problem}

The overall objective of assessing the impacts of climate change on the transport sector and the society in general, is to determine the optimal measures and strategies for eliminating or reducing their negative economic implications. This objective reveals the particularity of this problem; the goal is to plan an investment that does not aim to produce benefits but rather to reduce losses. Thus, the problem can be stated as follows: "Which are the optimal adaptation measures' combinations for achieving the maximum reductions (cost savings) of anticipated future losses on transport networks (and consequently to society) due to climate change?" This seemingly simple question conceals a large degree of complexity, because of its multidimensional character. In order to determine the optimal strategies, a sequence of successive and independent questions has to be answered, which together compose the entire problem. This series of questions describes the different dimensions of the problem that should be taken into 
account in the analysis. These questions, along with the respective dimensions, can be summarized as follows:

- Which are the vulnerability thresholds of transport networks? (this information is considered as known for the application of the proposed framework)

- Which are the most severe extreme events that are likely to happen, given the associated uncertainty for the prediction of such events? (Event with associated probability)

- Where will these events take place and which areas are affected?

- Which are the transport networks that will be affected the most?

- Which are the socio-economic impacts due to affected transport networks?

- Which are the measures and actions needed for addressing these events?

- Which is the optimal combination of measures that should be taken for achieving the maximum reductions of losses on transport networks, given the uncertainty contained in the occurrence probability of an event, the area allocation and the socio-economical quantification of its impacts?

- Can the above be monitored, evaluated and provided as useful tools to support decision makers?

The above sequence of questions is depicted in Fig. 1, representing the integrated approach of the multidimensional problem.

\subsection{Setting the problem}

The final objective is to provide a mathematical formulation, capturing all problem parameters, namely the dimensions of the event, the location, the networks (of same or different modes) and finally the adaptation measures. The problem addressed in the current paper can be formulated as: "For a period of the next $\mathrm{N}$ years $(N=30,40$ or 50 years) with an available budget $\mathrm{M}$, allocate $\mathrm{M}$ in such a way, so as to achieve the optimal reduction of the impacts due to future extreme weather events".

The approach of the problem is based on basic principles of economics, according to which a certain amount of money (resources) should be allocated in an optimal way, in order to satisfy maximum needs. The perspective of this approach can be understood as the one of a central government or any other organization, which seeks to allocate the available resources for covering future risks in an optimal way. This problem definition and perspective constitutes the originality and usefulness of the proposed framework; neither does it try to match adaptation measures to extreme events nor does it attempt to determine costs associated with certain transport modes and extreme events. The framework aims to set the problem in a realistic basis, where the first and most important step is to determine the priorities of investing the available resources by taking into account the impacts of all possible events, in all areas, for all transport networks and modes and finally to reduce them by selecting the optimal adaptation measures.

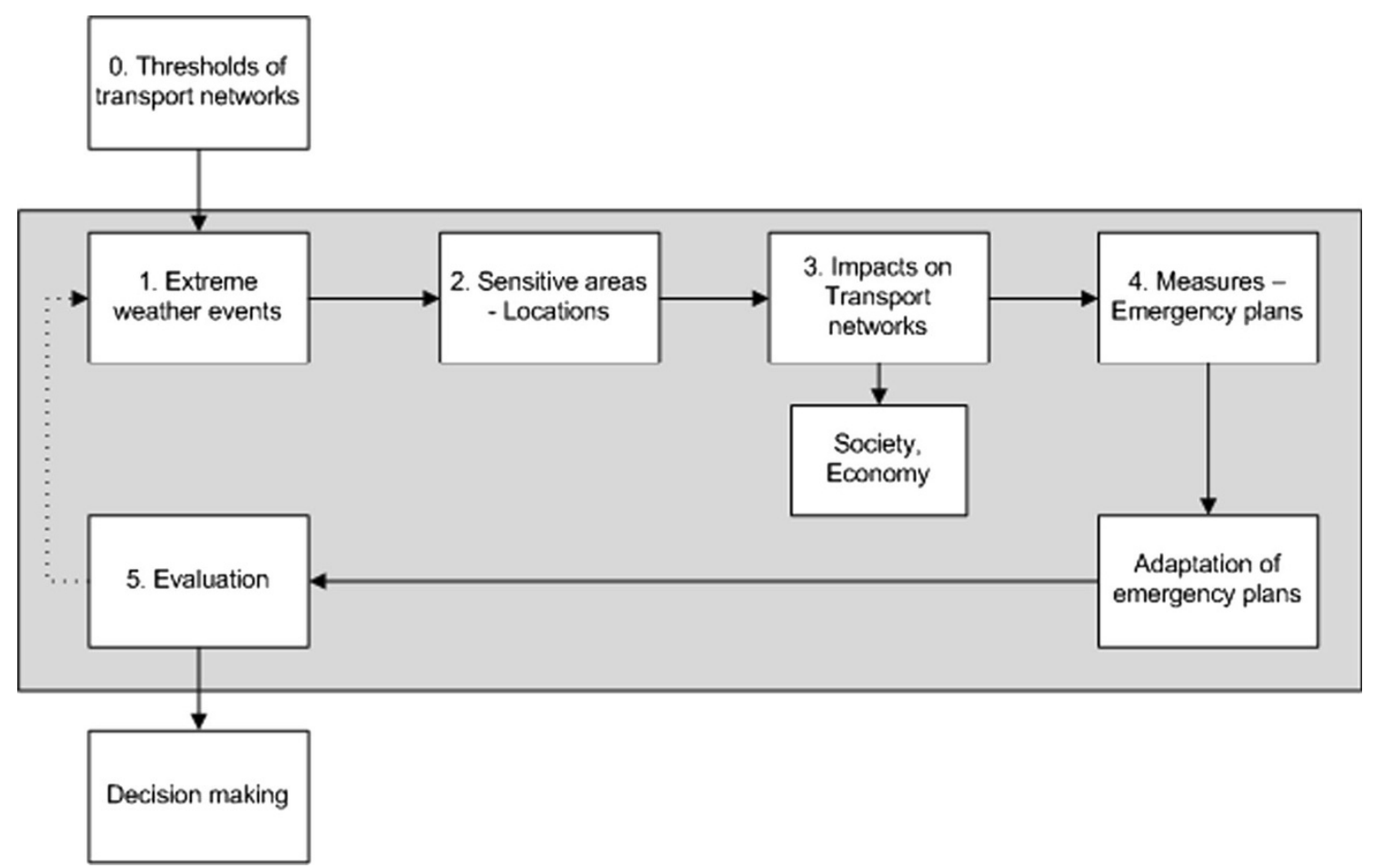

Fig. 1 Schematic representation of the integrated approach for assessing the impacts of climate change on transport networks 
It is noteworthy that vulnerability thresholds vary through time and thus the corresponding damage to infrastructure elements, such as a road link, is mediated by complex and dynamic factors, such as e.g. land use. However, the dynamic nature of these thresholds is not taken into account in the proposed framework, since it is assumed that the analysis described herein should be conducted instantly (without taking time into account) and in constant terms (updated regularly in a constant basis). A dynamic mathematical model for each transport network element would require a significant level of data collection and verification, which could result after a certain number of iterations of the 'instant' application of the framework.

\subsection{Basic definitions and assumptions}

It is important to provide a definition and differentiation between vulnerability and criticality. Herein, vulnerability of a network element is defined as its physical sensitivity to extreme weather events. Vulnerability is further associated to specific thresholds of extreme events' intensity, which may cause various levels of damages to transport infrastructures. Criticality of a network element, on the other hand, is a term associated to the entire network and is herein defined as the importance of the specific element in the network's regular operation. A necessary precondition for the application of the framework is that specific thresholds for the vulnerability of transport infrastructures are determined, as trigger points of different vulnerability levels. In this way, for each type of network infrastructure (road, rail etc.), all different levels of vulnerability are defined. This input is considered highly important, in order to compute the respective probabilities of each extreme event for all levels of intensity in the first step. Conclusively, as a starting point for the application of the framework, it is assumed that this information is known or given to the user of the proposed framework. It is apparent that different levels of costs are associated with varying vulnerability intervals. Herein, the uncertainty associated with the predictability of extreme weather events is considered as given and handled as the probability of an extreme weather event occurring.

\subsection{Identification of the common links for the analysis}

Figure 2 below contains the different dimensions of the problem, along with all the respective parameters (or levels) of each dimension that should be included in the methodological approach. In order to include all the different variables in a single top down approach, a 'reference unit' (common 'link') must be identified. This common 'link' should be capable of providing a unified categorization of different dimensions of the problem.
The most suitable parameter capable of providing a common classification framework is the 'area extent' of the extreme weather events. The categories of this specific dimension (local, regional, national and international) match with all the other dimensions and furthermore provide a sound theoretical basis for examining all the other dimensions of the problem. A more detailed explanation of each dimension linked to the 'area extent' is provided in Table 1.

Due to the above mentioned reasons, the area extent of an extreme weather event is the dimension that serves as the 'common link' in the analysis, providing a unified classification framework of all events, location of impacts, networks and emergency plans.

\section{Description of the framework}

\subsection{The 'event' dimension}

The first issue to be addressed before allocating an available budget $\mathrm{M}$ over a time-horizon $\mathrm{N}$, in order to reduce negative impacts of extreme weather events, is the accurate definition of the potential events during this specified period. An event $\mathrm{E}$ is defined as any extreme weather event or natural hazard that can cause malfunctions on transport networks (fires, floods, precipitation, hurricanes, storm surges, extreme heats, frost, landslides, wind and hail). In order to respond to an event in an optimal way, the exact impacts of the event must be predicted. The parameters that differentiate the impacts of an extreme weather event are:

- The frequency of the event (referring to the potential number of times that the event will occur during the defined period of analysis)

- The intensity of the event (referring to the way that every event is measured and to the respective thresholds associated to transport infrastructures). As an indicative example, one could mention that extreme heats can be measured in Celsius or Fahrenheit degrees, using three indicative categories: $40^{\circ}-45^{\circ}, 45^{\circ}-50^{\circ}$ and $>50^{\circ} \mathrm{C}$.

The output of this step is the probability of each event $\left(\mathrm{P}_{\mathrm{E}}\right)$ occurring in the period of analysis. The probability of each event is associated with the frequency, as well as with the intensity of the event.

\subsection{The 'area extent' dimension}

The area extent dimension reveals the area in which the event both takes place and affects. In the first case, it refers to the area whose infrastructure is affected (direct impacts due to the event), while in the second case it refers to the area whose 


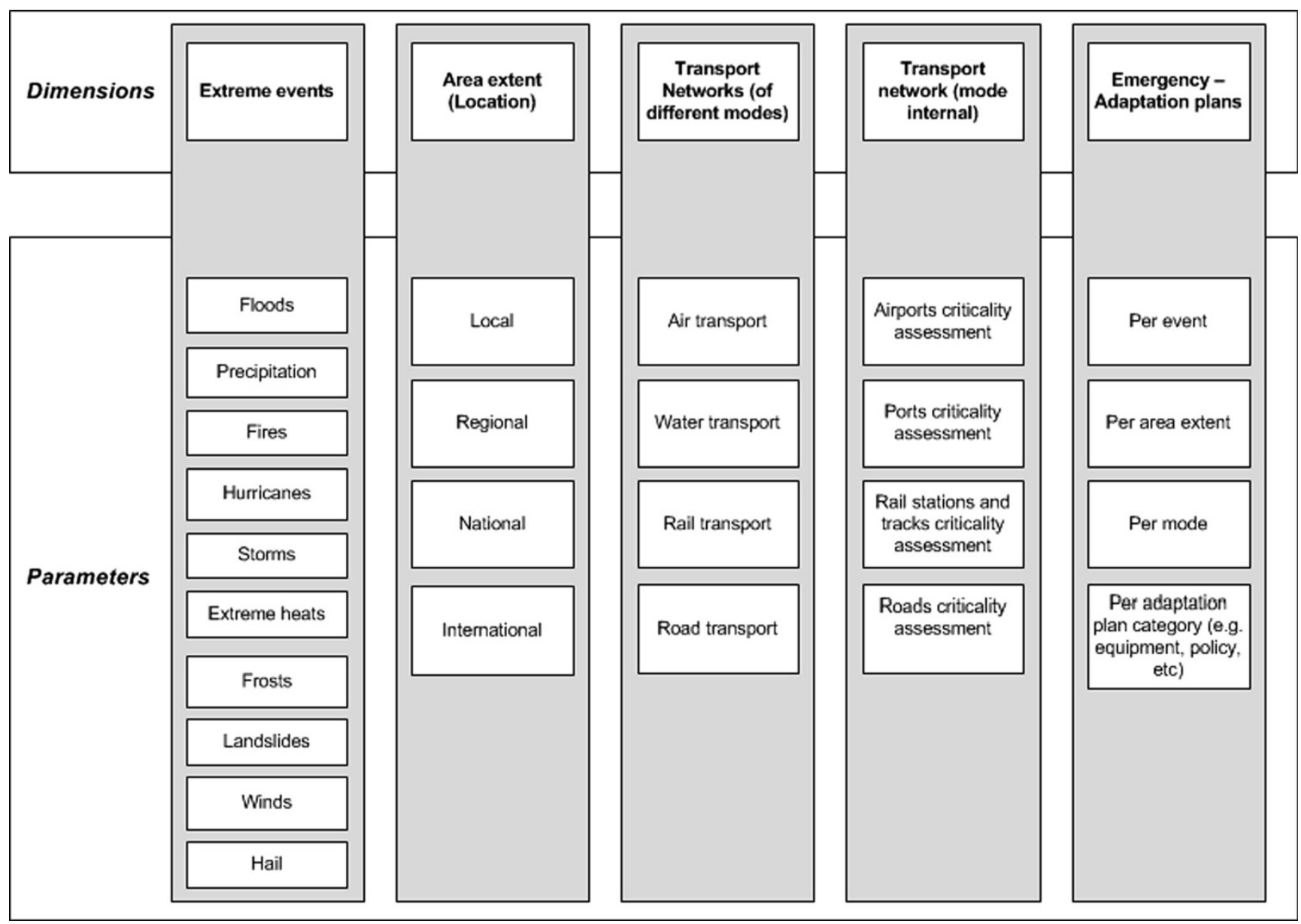

Fig. 2 Dimensions and parameters of the integrated framework

performance is affected (due to diverted traffic originating from closed links - indirect impacts). Thus, the dimension of 'location' is defined as the area whose transport networks will be affected by an event, resulting in any kind of infrastructure

Table 1 Linking 'Area extent' to the problem 'Dimensions'

$\begin{array}{ll}\begin{array}{l}\text { Problem } \\ \text { 'Dimension' }\end{array} & \begin{array}{l}\text { Link to 'Area extent' } \\ \text { Extreme Events }\end{array} \\ \begin{array}{c}\text { Their impacts can be categorized based on the } \\ \text { extent of the area }\end{array} \\ \text { Networks } & \begin{array}{l}\text { The different transport modes can be considered for } \\ \text { each specific area extent. The criticality } \\ \text { assessment methodology can also be defined, } \\ \text { considering different area scales. }\end{array} \\ \begin{array}{c}\text { The emergency management is classified in relation } \\ \text { to the area extent of the events, as the } \\ \text { measures }\end{array} & \begin{array}{l}\text { corresponding public authority is in charge of the } \\ \text { operation and has the overall responsibility. } \\ \text { Moreover, the adaptation plans follow the } \\ \text { classification of the authority and so the } \\ \text { classification of public authorities is based on the } \\ \text { 'area extent' of their jurisdiction. }\end{array}\end{array}$

oversaturation (due to diverted traffic caused by network failures), closures, malfunctions and damages. The classification of the location is described as the reference parameter for categorizing all the dimensions and parameters of the problem. A more thorough explanation of the different location types is provided below:

- Local level: An event that occurs at a local level indicates that its impacts are restricted in one city, one village or in general in a place that includes only one local public authority (e.g. a municipality is considered to belong at the 'local level').

- Regional level: An event that occurs at a regional level indicates that the impacts' area of the event covers more than one local public authority but in the same time the event is not a national scale emergency. Superior public authorities are responsible for the management of such events (e.g. prefectures or regional governments).

- National level: In this case the impacts of the event cover the entire (or almost entire) area of a country. The networks affected by the event influence transport networks and operations of the entire country to a certain degree. 
- International level: This is the highest level of 'area extent'. In this case the impacts of an event exceed the boundaries of one country.

The classification conducted in this step of the analysis is of a qualitative nature; it does not entail additional mathematical restrictions for the determination of the optimal adaptation strategies, but rather divides the whole process into four distinct categories, one for each type of area extent. This fact implies that in order to determine the optimal adaptation measures, the integrated methodology has to be implemented four separate times (stages). In the end, the outputs of each stage of the analysis will be overall evaluated for the assessment of the optimal adaptation plans.

\subsection{The 'network' dimension}

\subsubsection{The two phases of the network dimension}

This step is comprised by two phases; in the first phase an initial distinction is made between the different transport modes, while in the second phase the most critical components of each transport network have to be assessed for the interventions' priority setting.

\subsubsection{Criticality assessment for different transport networks}

The output of the first phase depends on the 'economic importance' of the respective modes for a specific area extent. The 'economic importance' of the different transport modes is defined as the total economic production quantity that is created from the regular function of each transport infrastructure. In order to optimally allocate a certain amount of money (resources) to different transport modes, the relative portion of the economic product of each transport mode is computed. The amount assigned to each transport mode is equal to its relative portion of the total socio-economic benefit of transport infrastructures for each area extent. Different socioeconomic benefits of individual modes (e.g. road, rail, maritime, air and others) can be expressed in monetary terms as a, $\mathrm{b}, \mathrm{c}, \mathrm{d}$ and $\mathrm{e}$, while the relative portion $\left(\mathrm{RP}_{\mathrm{i}}\right)$ per transport mode is equal to:

$R P_{i}=\frac{i}{\sum B=a+b+c+d+e}$

where $i=\{a, b, c, d, e\}$ and $\mathrm{B}=$ benefit

\subsubsection{Mode-internal criticality assessment}

There are three different criticality assessment approaches considered for the needs of the current paper, in relation to transport infrastructures; the link, the node and the combined link-and-node criticality assessment, each one associated with a specific transport infrastructure. It is important to mention, that due to factors such as land use and case specific special social needs (e.g. isolated islands) the following steps cannot be applied to local networks. For all other types of area extent, the methodology has to be implemented independently for each area extent.

The 'link' criticality assessment The link criticality assessment is applicable to road transport networks, in order to determine the most important links related to the efficiency of the network. The resulting importance - in the form of an index - for all network links is considered in the computation of the social costs. The steps of the respective method proposed to be used are presented next.

Step 1: The Origin-destination (OD) demand matrices are assigned on the road network, using given network data (ODs, centroids, connectors, links, and nodes). OD matrices are assigned in the network according to a user defined criterion (user equilibrium -UE, system optimum -SO).

Step 2: Network efficiency is computed, based on the Unified Network Performance Measure [20]:

$\varepsilon=\varepsilon(G, d)=\frac{\sum_{w \in W} \frac{d_{w}}{\lambda_{w}}}{n_{W}}$

where

$\varepsilon \quad$ unified network performance measure

$\mathrm{G} \quad$ network topology (links, nodes)

d demand vector (OD pairs)

W set of OD pairs

$\mathrm{d}_{\mathrm{w}} \quad$ demand of OD pair $\mathrm{w}$

$\lambda_{\mathrm{w}} \quad$ disutility of OD pair w (travel time)

$\mathrm{n}_{\mathrm{W}} \quad$ number of $\mathrm{OD}$ pairs for $\mathrm{G}$

Step 3: One link of the network is removed and $\varepsilon$ is computed again. Iteratively, this process is repeated for each link of the network in order to compute $\varepsilon$ for each removed link.

Step 4: The importance of each network component (link) is computed, based on the Network Component Importance [20]

$$
I(g)=\frac{\Delta \varepsilon}{\varepsilon}=\frac{\varepsilon(G, d)-\varepsilon(G-g, d)}{\varepsilon(G, d)}
$$

where $\mathrm{G}-\mathrm{g}$ is the resulting network after component $\mathrm{g}$ (link) is removed from network $\mathrm{G}$. 
Step 5: If the removal results in no path connecting an OD pair, the demand for that OD pair is assigned to an abstract path with a cost of infinity.

It is noted, that according to the definition of criticality assessment, a link is identified as critical, when there are limited or no available options serving the demand of OD pairs through this link [17], which in turn suggests that the costs for choosing an alternative path become high in comparison to the initial choice.

The 'node' criticality assessment The node criticality assessment is applicable to air and maritime transport networks. Airports and ports belonging to a certain area extent are ranked according to an index $\mathrm{V}_{\mathrm{i}}$ of the total value of their transport operations (e.g. on an annual basis). More specific, this index is defined as the relative value $\left(R V_{i}\right)$ per transport infrastructure in relation to the total value of all transport infrastructures of the same type (airports or ports) for a given area extent and it is equal to:

$R V_{i}=\frac{V_{i}}{\sum V_{i}}$

where $V_{i}=$ value of transport operations for each transport infrastructure.

The 'combined link-and-node' criticality assessment The combined link-and-node criticality assessment is applicable to rail transport networks. For the case of affected railway tracks only, the method to be applied is the same as for the link criticality assessment, with the assigned volumes corresponding to passenger trips. For the case of railway stations, the method to be applied is the modified link criticality assessment, where the criticality index of each node (railway station) is computed by removing simultaneously the links entering and exiting that node.

\subsection{The 'adaptation measures' dimension}

The proposed framework defines adaptation measures as investment combinations of different adaptation measures groups, associated to respective impacts of extreme weather events. Specifically, having a total budget $M$, this can be allocated to different combinations of adaptation measures $\bar{C}_{n}$. The aim of each combination $\bar{C}_{n}$ is to minimize the impacts of specific extreme weather events. The final objective is to optimally allocate the available sources for investment $\mathrm{M}$ (budget), so as to minimize the economic losses due to the impact of extreme events.

\subsection{Evaluation framework}

An effort to present the proposed method as a mathematical formulation is presented herein. The objective is to determine the appropriate measures' combinations $\bar{C}_{n}$ based on a finite budget $\mathrm{M}$, so as to minimize the economic losses due to the impact of extreme events for each specific area extent:

$\bar{C}_{n}(\mathrm{M}) \rightarrow \arg \min \sum\left(\mathrm{P}_{\mathrm{E}_{\mathrm{i}}} * \mathrm{~S}_{\mathrm{E}_{\mathrm{i}}}\right)$

where:

$\bar{C}_{n} \quad$ set of measures

$\mathrm{M} \quad$ available budget (finite)

$\mathrm{P}_{\mathrm{E}} * \mathrm{~S}_{\mathrm{E}}$ impact

$\mathrm{E}_{\mathrm{i}} \quad$ events $\mathrm{P}_{\mathrm{E}}=$ probability of an event $\mathrm{E}$ occurring

$\mathrm{S}_{\mathrm{E}} \quad$ severity of an event $\mathrm{E}$

$\mathrm{S}_{\mathrm{E}} \quad \mathrm{S}_{\mathrm{EI}}+\mathrm{S}_{\mathrm{ES}}$

$\mathrm{S}_{\mathrm{EI}} \quad$ damage and rehabilitation costs

$\mathrm{S}_{\mathrm{ES}} \quad \mathrm{t}_{\mathrm{d}} * \mathrm{~d} * \mathrm{vt}$ (societal costs)

$t_{d} \quad$ delay

d demand

vt average value of time (of passenger and freight transport)

The appropriate measures' combinations are determined according to the outputs of the criticality assessment.

\section{Conclusions}

Following a targeted literature review aiming to highlight the existing research and methodological gaps in the assessment of climate change impacts on transport networks, the present paper describes an integrated approach, which can effectively address, in a sequential way, all the major dimensions of the problem. The area extent has been identified as the only common parameter, which can provide a classification framework for the remaining problem dimensions and respective parameters. The proposed framework provides a top-down approach from the central government perspective, addressing real world questions and issues about determining the appropriate combination of measures, in order to achieve the minimization of the economic losses due to potential extreme weather events with limited available resources. A major further step of the current research includes the examination of an actual implementation of the proposed framework, based on specific extreme weather events, actual transport networks, the assessment of the framework's outputs and their usability by decision makers.

Acknowledgements The research presented in the present paper has been conducted within the context of the WEATHER project "Weather Extremes: Assessment of Impacts on Transport Systems and Hazards for 
European Regions", funded by the Research Directorate General of the European Union under the Seventh Framework Programme for Research and Technological Development.

Open Access This article is distributed under the terms of the Creative Commons Attribution License which permits any use, distribution, and reproduction in any medium, provided the original author(s) and the source are credited.

\section{References}

1. White Paper 'Adapting to climate change: Towards a European framework for action', COM (2009) 147 Final. Commission of the European Communities. Brussels, 2009

2. Commission staff working document 'Climate Change and Water, Coasts and Marine Issues', SEC (2009) 386. Commission of the European Communities. Brussels, 2009

3. Commission staff working document 'Impact Assessment', SEC (2009) 387. Commission of the European Communities, Brussels, 2009. Change. http://www.ipcc.ch/ipccreports/assessments-reports.htm

4. OECD (2008) 'Economic Aspects of Adaptation to Climate Change Costs Benefits and Policy Instruments', http://www.oecd.org/env/cc/ adaptation

5. Hamin EM, Gurran N (2009) Urban form and climate change: Balancing adaptation and mitigation in the U.S. and Australia. Habitat Int 33:238-245

6. Hunt A, Watkiss P (2011) Climate change impacts and adaptation in cities: a review of the literature. Clim Chang 104:13-49

7. Love G, Soares A, Puempel H (2010) Climate change, climate vulnerability and transportation. Procedia Environ Sci 1:130-145

8. Knoop VL, Snelder M, van Zuylen H, Hoogendoorn S (2012) Linklevel vulnerability indicators for real-world networks. Transp Res Part A 46:843-854

9. Taylor MAP, D’Este GM (2007) Transport Network Vulnerability: a Method for Diagnosis of Critical Locations in Transport Infrastructure Systems. Critical Infrastructure: Reliability and Vulnerability, pp. 9-30

10. Jenelius E (2007) Incorporating dynamics and information in a consequence model for road network vulnerability analysis. In: Van Zuylen HJ (ed) Proceedings of Third International Symposium on Transport Network Reliability. The Hague, The Netherlands

11. Knoop VL, Hoogendoorn SP, Van Zuylen HJ (2008) The influence of spillback modeling when assessing consequences of blockings in a road network. Eur J Transp Infrastruct Res 8(4): 287-300

12. Taylor MAP, Sehkar SVC, D'Este GM (2006) Application of accessibility based methods for vulnerability analysis of strategic road networks. Networks Spat Econ 6:267-291
13. Jenelius E, Petersen T, Mattsson L-G (2006) Importance and exposure in road network vulnerability analysis. Transp Res Part A 40:537-560

14. Sohn J (2006) Evaluating the significance of highway network links under the flood damage: an accessibility approach. Transp Res Part A 40:491-506

15. Erath A, Birdsall J, Axhausen KW, Hajdin R (2010) Vulnerability assessment methodology for Swiss road network. Transp Res Rec 2137:118-126

16. Kim TJ, Lee JS (2006) Development of Index for Identifying Critical Transportation Links. In: Irene J (ed) Uddevalla Symposium: Entrepreneurship and Development-Local Processes and Global Patterns. University West, Trollhättan

17. Nagurney A, Qiang Q (2008) A network efficiency measure with application to critical infrastructure networks. J Glob Optim 40:261275

18. Schulz C (2007) Identification of critical transport infrastructures, Climate change and vulnerability: challenges for spatial planning and civil protection

19. Murray-Tuite PM, Mahmassani HS (2004) Methodology for determining vulnerable links in a transportation network. Transp Res Rec 1882:88-96

20. Chen A, Yang C, Kongsomsaksakul S, Lee M (2007) Network-based accessibility measures for vulnerability analysis of degradable transportation networks. Networks Spat Econ 7:241-256

21. Sullivan JL, Novak DC, Aultman-Hall L, Scott DM (2010) Identifying critical road segments and measuring system-wide robustness in transportation networks with isolating links: a link-based capacity-reduction approach. Transp Res Part A 44:323-336

22. Eichhorst U (2009) Adapting Urban Transport to Climate Change. Sustainable Transport: A Sourcebook for Policy-makers in Developing Cities

23. Suarez P, Anderson W, Mahal V, Lakshmanan TR (2005) Impacts of flooding and climate change on urban transportation: a systemwide performance assessment of the Boston metro area. Transp Res Part D 10:231-244

24. Koetse MJ, Rietveld P (2009) The impact of climate change and weather on transport: an overview of empirical findings. Transp Res Part D 14:205-221

25. EMA-Emergency Management in Australia, Concepts and Principles (2004) Australian Government

26. FEMA - Federal Emergency Management Agency, Principles of EM' (2007) http://www.iaem.com/publications/documents/ PrinciplesofEmergencyManagement.pdf

27. Jaroszweski D, Chapman L, Petts J (2010) Assessing the potential impact of climate change on transportation: the need for an interdisciplinary approach. J Transp Geogr 18(2):331-335 